\title{
Countermovement Jump with the Help of Wings in 16-Year- old Volleyball Players
}

\author{
Junida Pogoni \\ Artan Pogoni \\ PhD, Sports University of Tirana, Faculty of Physical \\ Activity and Recreation, Department of Movement and Health
}

\section{Abstract}

Vertical jump is combined with the assessment of the strength and strength of the muscles of the lower extremities that are fundamental components of the volleyball game's work.The Countermovement Jump with arm (CMJA) test as a specific specifically for the assessment of the offensive force of volleyball players.Methods: Female (F) \& Males (M) average volleyball players were tested in CMJA at the GFRP; Force(F max), Power(P max) and gravity shift relationship Jump Height(JH). Anthropometric measurements of volleyball players were also developed; Body Height $(\mathrm{BH} \mathrm{cm})$, Body Mass (BMI\% $\mathrm{kg} / \mathrm{m}^{2}$ ), Body Weight (BW kg). Results: The differences between the two groups found in the study resulted in significant differences in BH cm (F-172, $\mathrm{M}-187.3$ ), BW $\mathrm{kg}$ (F- 62.2, M -79.3), BMI kg / $\mathrm{m}^{2}$ (F-21.1, M -22.6), Jump place in JP cm (F-266, M-310), Jump Attack in JA cm (F-274, M-321). But even the data captured by the Leonardo platform in the CMJA test gives us a noticeable difference between the two groups; (F-6.4,M-4.5), P max (F-3.08, M-4.78) and diff P max Left \& Right(F-2.59,M-0.56),V max m/\%(F-5.2,M-4.4). Conclusion: The results obtained suggest changes to the performance of volleyball players in "vertical jump" in the parameters of force, speed, and power. These indicators are valid for any trainer or volleyball player to implement a detailed and specific training program for the further development of the physical qualities of volleyball players, especially vertical tipping in gaining the lower extremity muscular power.

Keywords: volleyball, jump, players, countermovement

\section{Introduction}

The studies that I've done have consistently been the basis for the volleyball sport in the new age of 16-17years old. Achieving high levels of modernity requires a great deal of study, a research work to develop and perfect physical performance in professional sports. The important tendency in world volleyball, in addition to 
improving the body height of players, is the increase in the vertical jump level. In collective sports as volleyball, football, basketball besides other technical elements that these kind of sports contain it is seen even the functioning of the element "vertical jump". The best perfection of a vertical jump is achieved with a purposeful training to increase the jump height, the high degree of muscular activity achieved with a specific training program. Plyometric exercises use the stretch-shorten cycle to train the muscles to perform and perform greater work in the shortest possible time (9). Plyometric is a form of resistance exercise that refers to the stretch-shortening cycle (SSC) such as jumps or doing vertical or horizontal jumps (10). With vertical jump, we understand the athlete's ability to elevate his body's gravity center with the help of dynamic muscular work of lower extremities. Physiological studies on this discipline have shown how a volleyball player should possess and increase, through training, the ability to develop explosive strength and to reuse elastic energy. Any physical exercises, they may be defined by means of the volume expressed by work performed during jumps (2) or by the number of jumps (3) as well as by intensity, which is usually defined by such parameters as ground reaction forces (GRFP) and the rate of force development $(4,5)$. The main purpose of this study is to evaluate the changes and the effects that training in volleyball player's female \& male using the tests countermovement jump with arm (CMJA).

\section{Methodology}

The subjects of this study were 12 Female \& 12 Male volleyball player participating in the championship of volleyball in Albania. The main objective is to see to these volleyball players aged 16 years old differences in changes in the measurements of some functional parameters. It will be possible to see the state of the level of volleyball player's in our country through measurement and data analysis.

Table.1. Anthropometric measurements volleyball players.

\begin{tabular}{|c|c|c|}
\hline Groups & FEMALE(F) & MALE(M) \\
\hline Mean Age & Age & Age \\
\hline Body Height & $(\mathrm{BH}) \mathrm{cm}$ & $(\mathrm{BH}) \mathrm{cm}$ \\
\hline Body Wight & $(\mathrm{BW}) \mathrm{kg}$ & $(\mathrm{BW}) \mathrm{kg}$ \\
\hline Body Mass & $\mathrm{BMI} \% \mathrm{~kg} / \mathrm{m}^{2}$ & $\mathrm{BMI} \% \mathrm{~kg} / \mathrm{m}^{2}$ \\
\hline Jump Place & $\mathrm{JP}$ & $\mathrm{JP}$ \\
\hline Jump Attack & $\mathrm{JA}$ & $\mathrm{JA}$ \\
\hline
\end{tabular}

\subsection{Protocols of the Test Performed}

Tests protocol that we used in this experiment was designed to assess the capacity of all parameters of vertical jump height. These tests called Bosco tests describe the method used to measure the power, the strength of the lower extremities. Tests are 
performed on laboratory equipment platform Leonardo® system Ground Reaction Force Plate (GRFP) .Countermovement jump test with the help of Arm- CMJA; Jump against action (move) without the aid of the arms CMJ, but the athlete begins testing position at attention and takes off refractive limbs bottom up to $90^{\circ}$ with the help of the arm to pick up momentum to jump in platform GRFP. This test assesses the strength of the energy reuse explosive elastic (6). The use of wings in the vertical jump indicates the duration of an impulse.

\section{Results}

The following table 2 and graphic 1 presents the average data of the two groups from field measurements. There are visible differences between the sexes.

Tab, 2. Measurements volleyball players in ground

\begin{tabular}{|c|c|c|c|c|c|c|c|}
\hline $\mathrm{Nr}$ & Group & Age & $\begin{array}{c}\mathrm{BH} \\
\mathrm{cm}\end{array}$ & $\begin{array}{c}\mathrm{BW} \\
\mathrm{kg}\end{array}$ & $\begin{array}{c}\mathrm{BM} \\
\mathrm{Kg} / \mathrm{m}^{2}\end{array}$ & $\begin{array}{c}\text { Jump } \\
\text { Place } \\
\mathrm{cm}\end{array}$ & $\begin{array}{c}\text { Jump } \\
\text { Attack } \\
\mathrm{cm}\end{array}$ \\
\hline 12 & FEMALE & 16.9 & 172 & 62.2 & 21.1 & 266 & 274 \\
\hline 12 & MALE & 16.8 & 187.3 & 79.3 & 22.6 & 310 & 321 \\
\hline
\end{tabular}

Graphic.1. Measurements volleyball players.

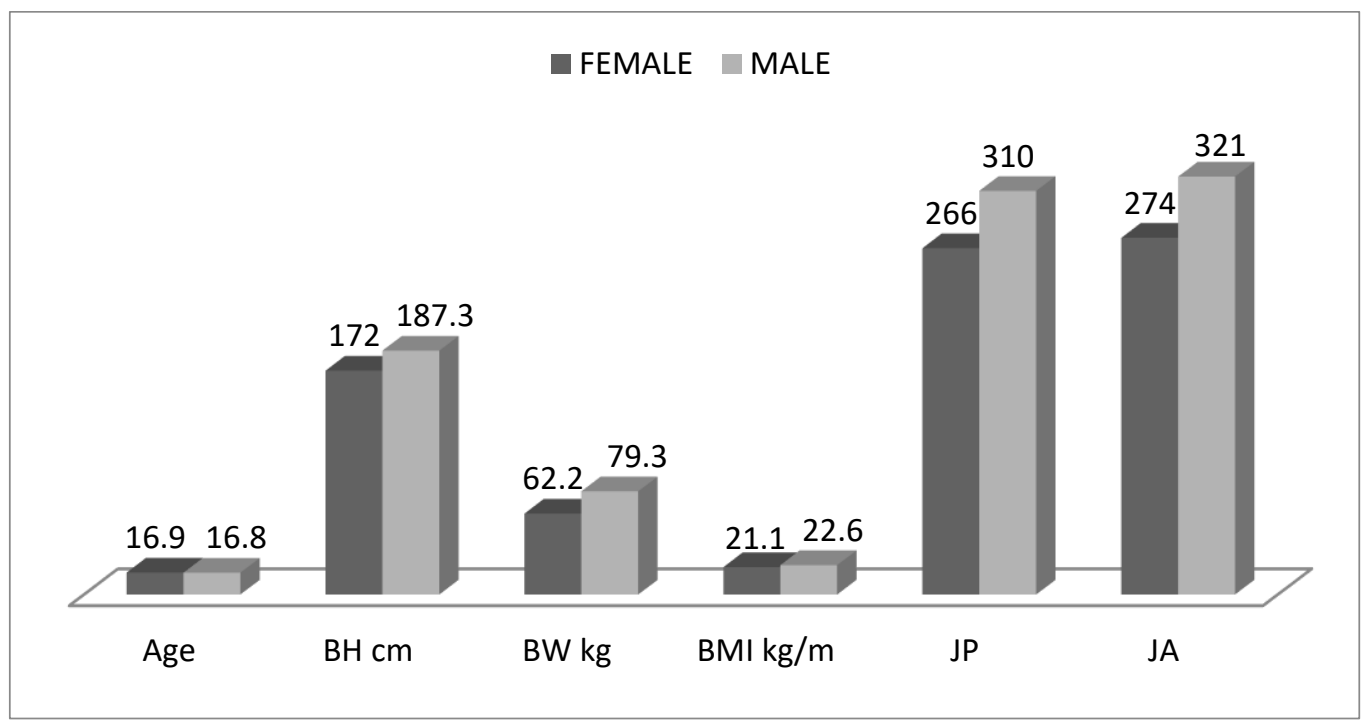

The results obtained from the GRFP platform of the specific tests developed by the groups given in the study will be presented below in separate tables 3 and graphic 2 . The average values of the groups taken in the anti-action test of the CMJA are presented. 
Tab, 3. Measurements CMJA tests in "Leonardo" platform Female\&Male

\begin{tabular}{|c|c|c|c|c|c|c|c|}
\hline Nr & Group & $\begin{array}{c}\text { Jump } \\
\text { Height m }\end{array}$ & $\begin{array}{c}\text { Vmax } \\
\mathrm{m} / \mathrm{s}\end{array}$ & $\begin{array}{c}\text { Fmax } \\
\mathrm{kn}\end{array}$ & $\begin{array}{c}\text { diff.Fmax } \\
\text { Left-Right\% }\end{array}$ & $\begin{array}{c}\text { Pmax } \\
\mathrm{kw}\end{array}$ & $\begin{array}{c}\text { diff.Pmax } \\
\text { Left- } \\
\text { Right } \\
\%\end{array}$ \\
\hline 12 & FEMALE & 0.45 & 2.59 & 1.42 & 6.4 & 3.08 & 5.2 \\
\hline & Max & 0.98 & 6.4 & 2.49 & 4.78 & 2.36 & 2.43 \\
\hline & Min & 0.72 & 4.05 & 2.34 & 3.08 & 1.53 & 1.55 \\
\hline 12 & MALE & 0.56 & 2.95 & 1.93 & 4.05 & 4.78 & 4.42 \\
\hline & Max & 0.98 & 6.4 & 2.49 & 6.4 & 3.08 & 5.2 \\
\hline & Mini & 0.45 & 2.59 & 1.42 & 3.08 & 1.53 & 1.55 \\
\hline
\end{tabular}

Graphic 2.Measurements CMJA tests in "Leonardo" platform Female\&Mal

\section{FEMALE MALE}

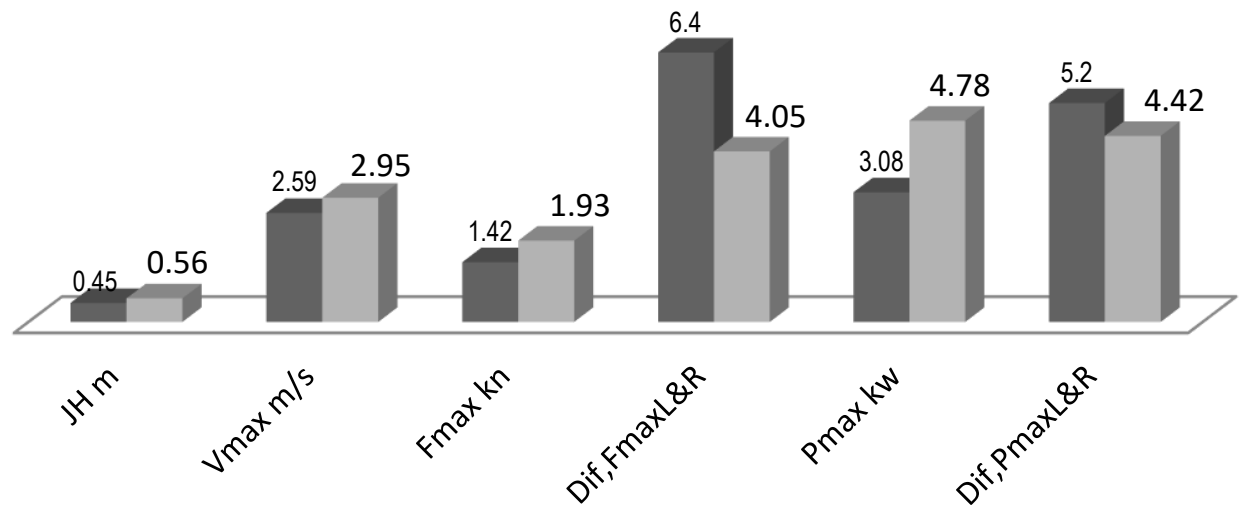

\section{Analyses}

Max force (F max) and max power (P max) are relatively poor values (low) compared with the results that a volleyball player should have during a vertical jump.The table shows the data of Male \&Female volleyball players in the tests done in "Leonardo" platform and the average data of the two groups from field measurements.. Volleyball players according to a study [1,44-45] reports that the vertical jump height is within the range of $75-90 \mathrm{~cm}$ which reflects the fact that the excessive increase of the maximum strength of the lower extremities results in a reduction in the speed of the movement, namely the reduction the level of volleyball dance from the 
ground.Specifically from what is presented above in Table 3 of the descriptive analysis values obtained from GFRP in Jump height Female $0.45<$ Male 0.56 , Vmax was detected Female $2.59<$ Male 2.95, Fmax was detected for Female $1.42<$ Male 1.93 $\mathrm{kN}$. The left and right footing differences on the leftforms were for Female 6.4\%> Male $4.05 \%$. The power ratings obtained on the platform are Female $3.08<$ Male 4.78 and the differences between left and right foot Female 5.2\%> Male $4.42 \%$. From the control of difference averages results that women do not have a good development of vertical dance performance through the CMJA test. The t-test result shows that the differences between the two groups were statistically significant ( $\operatorname{sig}=0.035$ ).

\section{Discussion}

Many researchers and practitioners are encouraged to consider this methodology and these variables as valuable and reliable measures to determine athletes' ability to perform in vertical dance. The main purpose of the CMJA test at volleyball players of these new age groups was to determine the reliability measurement of the main performance measures commonly used to determine the CMJ strength capacity qualities from GRFP data.. The long-term CMJ test is supposed to provide a measure of muscle elasticity, whereas today it is found that this test provides the mass of rapid jump force (7)where the differences between the CMJ and SJ tests proposed the "elasticity "Of athletes in the team using the concept" elasticity index "coming from the difference of these two tests. The good capacity used by elastic energy corresponds to $8-10 \mathrm{~cm}$. The difference between these two tests is called the fast power index. The elasticity coefficient of formula (CMJ-SJ) x100 / CMJ expressed in\% is an index of accumulated energy capacity as a result of elastic muscle extension preceding muscular contraction and meanwhile from the data presented to the team Volleyball in Albania has reported a low percentage of elastic energy as a result of a poorly recommended exercise to develop this quality. The vertical jump performed by the two study groups in the CMJA test that provides the mass of the rapid jump force has been shown that volleyball players with these values are at the levels of the capacity of the low-speed force. A comparison of body height during CMJ and body weight also allows the assessment of the start of the test threshold coordination and the end of the jump stage according to GRFP ( $<<0.01$, force $p \leq 0.05)$, CMJ ( $p<0.01$, force $\mathrm{p}<\mathrm{p}$ 0.01), for 8 weeks (8).

\section{Conclusion}

The team's female \& male in the study had significant differences between them in the technical element of jumping and its processing. The data from this study will be available for coaches who want to improve their vertical jump performance to players. The ability to jump is an important factor of success in this sport, but it is associated with the general and specified training for the development of its performance regardless of gender players. Interpretation of the data suggests that young volleyball players are indispensable to apply the exercises that improve the dance performance as an important element in this sport. The results of this study 
illustrate the importance of recognizing the impact of rapid force, emphasizing the need to understand that specific assessment techniques that will identify in individual components the strength and strength qualities that affect the performance of the jump.

\section{References}

[1] Bosco,C., Komi PV. (1981).Influence of countermovement amplitude in potentiation of muscular performance. In: Biomechanics VII-A. Morecki A, Fidelis K, Kedzior K, Wit A, eds. Baltimore, MA: University Park, 129- 135.

[2] Chelly, MS, Hermassi, S, Aouadi, R, dhe Shephard, 2014. Effects of 8-week plyometric season on the progress of upper and lower limbs adolescent elite handball players. RJ Forca J Cond Res 28 (5): 1401-1410.

[3] De Villarreal E.S., Kellis E., Kreamer W.J., Izquierdo M. Determining variables of plyometric training for improving vertical jump height performance: a meta-analysis. J. Strength Cond. Res. 2009;23:495-506pp.

[4] Fleck, S. J., \& Kraemer, W. J. (2004). Advanced training strategies. In S. J. Fleck \& W. J. Kraemer (Eds.), Designing Resistance Training Programs (3rd ed., pp.209-239). Champaign, IL: Human Kinetics.

[5] Gilles Cometti,Dominique Cometti;"La Pliometria(origini,teorie,allenamento) 2a edizione italiana (Tivoli 2009)Capitolo V; I TEST. Test di Bosco fq60-78.

[6] Holcomb William R.; Lander, Jeffrey E.; Rutland, Rodney M.; Wilson, G. Dennis. Journal of Strength and Conditioning Research: May 1996 - p 89-92 .

[7] Jensen R.J., Ebben W.P. Quantifying plyometric intensity via rate of force development, knee joint, and ground reaction forces. J. Strength Cond. Res. 2007;21:763-767pp.

[8] Lin J., Chao Ch., Liu Y. The comparison of work intensity and exercise performance between short-stretch and long-stretch drop jump. In: Y. Hong, D.P. Johns, R. Sanders (eds.) Proceedings of the 18th International Symposium on Biomechanics in Sports. Hong Kong, China: International Society of Biomechanics in Sports Newsletter. Hong Kong 2000;pp.58-61pp.

[9] V.Rizvanolli. 1997. Kërcimi në Volejboll. Fq.44.

[10] Walsh M., Arampatzis A., Schade F., Brüggeman G.P. The effect of drop jump starting height and contact time on power, work performed and moment of force. J. Strength Cond. Res. 2004;18: 561-566pp. 